MKG-Chirurg 2011 · 4:146-147

DOI 10.1007/s12285-011-0213-7

Online publiziert: 1. September 2011

(c) Springer-Verlag 2011

\author{
K. Wangerin ${ }^{1}$ - A.C. Kübler ${ }^{2}$ \\ ${ }^{1}$ Klinik für Gesichts-, Kiefer- und Wiederherstellungschirurgie, \\ Paracelsus-Krankenhaus Ruit, Ostfildern \\ ${ }^{2}$ Klinik und Poliklinik für Mund-, Kiefer- und Plastische Gesichtschirurgie, \\ Universitätsklinikum Würzburg
}

\title{
Orthognathe Chirurgie
}

Sehr verehrte Frau Kollegin,

sehr geehrter Herr Kollege,

die operative Korrektur schwerer Bissanomalien hat in den letzten 40 Jahren eine fulminante Entwicklung genommen.

In den 7oer-Jahren des letzten Jahrhunderts wurden vorwiegend nur anteriore Segmentosteotomien mit gleichzeitigen Zahnextraktionen zur Rückverlagerung im anterioren Ober- und Unterkiefer durchgeführt. Offene Bisse wurden durch Intrusion von lateralen Oberkiefersegmenten korrigiert. In allen Fällen war ein längerer stationärer Aufenthalt mit Drahtverschnürung der Kiefer, die die postoperative Lebensqualität erheblich einschränkte, notwendig. Die vollständige Verlagerung des Unterkiefers wurde vorwiegend zur Korrektur der mandibulären Prognathie vorgenommen. Viel später, etwa Mitte der 8oer-Jahre wurde auch die LeFort-I-Osteotomie Routine. Diese Methode galt wegen der Gefahr eines unbeherrschbar hohen Blutverlusts lange als äußert gefährlich.

\section{- Erst die „Down-Fracture-Technik“ führte zu mehr chirurgischer Sicherheit und größeren Verlagerungsmöglichkeiten in alle Richtungen.}

In den 8oer-Jahren setzten sich in den deutschen kieferorthopädischen Praxen, aus USA kommend, festsitzende Multibandtechniken zur wirksameren Koordination der Zahnbögen durch. Dies führte zu einer Zunahme der interdisziplinären Zusammenarbeit mit den Kieferorthopäden, einer rapiden Abnahme der bisherigen Segmentosteotomien und zu einer
Zunahme von vollständigen Kieferverlagerungen. Die Übernahme von kephalometrischen Analysen aus der Kieferorthopädie zur Operationsplanung, die Operationssimulation an arbiträr einartikulierten Kiefermodellen mit Herstellung von Splinten und die Entwicklung von Miniplattenosteosynthesen zur übungsstabilen Kieferfixierung hatten Anfang der 9oerJahre mehr und mehr die simultane Verlagerung von Ober- und Unterkiefer zur Folge.

Die bignathe Osteotomie halbierte die Verlagerungsstrecke zur Beseitigung eines Fehlbisses pro Kiefer. Die Ergebnisse wurden unter gleichzeitiger kieferorthopädischer Behandlung stabiler. Allerdings war eine postoperative Intensivüberwachung von mehreren Tagen nötig, die Gabe von Eigenbluttransfusionen obligat, und die Verschnürung der Kiefer betrug zunächst noch mehrere Wochen, konnte aber bis zum Jahre 2000 in den meisten Kliniken Deutschlands auf etwa 1 Woche reduziert werden.

Mit der konsequenten Infiltrationsanästhesie der enoralen Operationszugänge mit Lokalanästhesie und Vasokonstringens und Einführung der kontrollierten Blutdrucksenkung konnten Bluttransfusionen in den ersten Jahren nach der Jahrtausendwende bis auf seltene Ausnahmen vermieden werden. Bignathe Osteotomien erfolgten nun ohne Immobilisation. Durch die chirurgische Sicherheit, schwere Bissanomalien verlässlich zu korrigieren, konnte sich das Augenmerk des Chirurgen mehr auf ästhetische Aspekte konzentrieren. So dienten kephalometrische Planungen jetzt zur Einstellung knöchernen Strukturen in Normposition, nicht nur zur Fehlbissbeseitigung; Kinn- und
Jochbeinkorrekturen erfolgten gleichzeitig. Viele Überlegungen konzentrierten sich auf die Normalisierung des Gesichtsprofils, den Nasolabialwinkel, die Frontzahnschau und das Lippenprofil. Die Beseitigung des schweren Fehlbisses wurde fast zum „Nebenprodukt“.

\section{- Heute gehen einer operativen Fehlbisskorrektur umfangreiche Planungen mit dem Kieferorthopäden voraus.}

In schweren Fällen erfolgen die Korrekturen zweizeitig, zuerst die transversale Oberkieferdistraktion oder die vertikale Ramusdistraktion. Bignathe Korrekturen werden danach oft mit Mehrteilungen des Oberkiefers oder Segmenten im Unterkiefer kombiniert. Osteosynthesen erfolgen inzwischen standardisiert in $\mathrm{Ab}$ hängigkeit von Ausmaß und Richtung der Verlagerung. Die Funktion der Nerven bei der Neurolyse überwiegend im Unterkiefer kann durch piezoelektrische Freilegungen in der Regel erhalten werden.

Sekundäre Korrekturen des Gesichtsweichgewebes einschließlich der abschließenden Nasenkorrektur werden routinemäßig mit der Entfernung der Osteosynthesematerialien verbunden. In zunehmendem Maße werden sogar Verlagerungen von Mittel- und Untergesicht vorgenommen - ohne Korrektur eines Fehlbisses, aus rein ästhetischen Gründen.

Liegt ein Syndrom vor, wird je nach medizinischer Notwendigkeit teilweise schon während des skeletalen Wachstums operiert. Die Eingriffe können später nach Wachstumsabschluss durchaus wiederholt werden. Die Komplexität dieser systematisierten Eingriffe bezieht in 
Kooperation mit Neurochirurgen teilweise die Frontobasis und sogar die gesamte Schädelkalotte mit ein.

Das Ausmaß skeletaler und weichgewebiger Korrekturmöglichkeiten des gesamten Gesichts und des Kopfes haben so sehr zugenommen, dass sie vornehmlich in interdisziplinären Zentren umfassend angeboten werden. In den Leitthemenbeiträgen werden aus den Zentren Würzburg und Stuttgart aktuelle chirurgische Konzepte dargestellt.

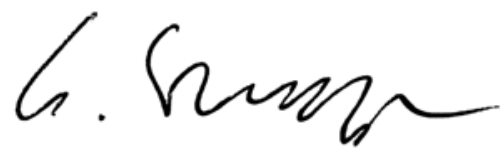

Prof. Dr. Dr. Dr. h. c. K. Wangerin

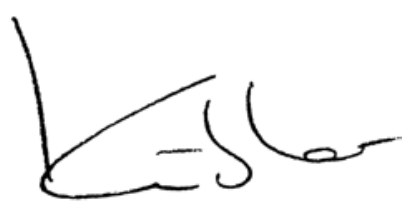

Prof. Dr. Dr. A.C. Kübler

\section{Korrespondenzadresse \\ Prof. Dr. Dr. Dr. h. c. K. Wangerin}

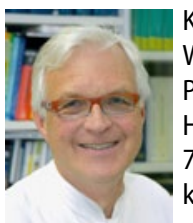

Klinik für Gesichts-, Kiefer- und Wiederherstellungschirurgie, Paracelsus-Krankenhaus Ruit Hedelfinger Str. 166, 73760 Ostfildern k.wangerin@kk-es.de

\section{Prof. Dr. Dr. A.C. Kübler}

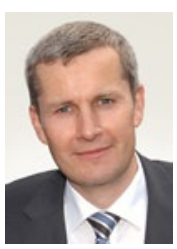

Klinik und Poliklinik für Mund-, Kiefer- und Plastische Gesichtschirurgie, Universitätsklinikum Würzburg Pleicherwall 2, 97070 Würzburg kuebler_a@

zahnklnik.uni-wuerzburg.de

\section{springerzahnmedizin.de Ihr Fortbildungsportal ist da!}

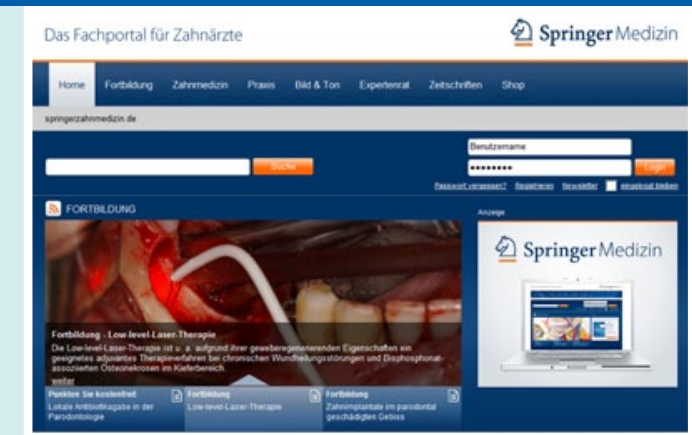

bietet mit interessanten allgemeinmedizinischen Beiträgen einen Blick über den Tellerrand. Unter dem Punkt „Praxis" verbergen sich verschiedenste Informationen und Tipps rund um den Praxisalltag, wie z.B. Mitarbeiterführung, Patientenkommunikation, Steuer- und Rechtstipps und vieles mehr. Unter „Zeitschriften" finden Sie die Archive der zahnmedi-

Sie möchten sich fortbilden und CMEPunkte sammeln? Oder einfach nur Ihr Fachwissen testen? Sie interessieren sich für konkrete Fallbeschreibungen in Form von Bilderstrecken oder möchten einen Experten Ihres Fachgebietes um Rat fragen? Gehen Sie online und entdecken Sie die vielfältigen Angebote von springerzahnmedizin.de, dem neuen Fortbildungsportal für Zahnmediziner und MKG-Chirurgen.
er-Fachzeitschriften. Eine zinischen Springer-Fachassende Suchfunktion bringt Sie schnel und sicher zu den verfügbaren Beiträgen, die Sie downloaden, abspeichern und ausdrucken oder sich einfach nur in Ihrer persönlichen Merkliste vormerken können. Ergänzt wird das Angebot durch zahlreiche anschauliche Bilderstrecken, interessantes Videomaterial und auch der fachliche Austausch kommt auf springerzahnmedizin.de nicht zu kurz: Stellen Sie dem „Expertenrat"

\section{Wählen Sie aus unseren über 100 Fortbildungen und seien Sie immer auf dem neuesten Stand.}

Speziell zugschnitten auf die Bedürfnisse von Zahnmedizinern und MKG-Chirurgen hat sich springerzahnmedizin.de in erster Linie der Fortbildung verschrieben. Unter dem entsprechenden Navigationspunkt finden Sie daher zahlreiche Fortbildungsbeiträge aus den deutschsprachigen zahnmedizinischen Fachzeitschriften von Springer Medizin. Sammeln Sie online CME-Punkte oder überprüfen Sie Ihr Wissen mit unseren Wissenschecks - bei springerzahnmedizin.de ist Fortbildung mehr als nur Punkte sammeln.

\section{Alles aus der Zahnmedizin und noch viel mehr}

Neben Inhalten aus allen Bereichen der Zahnmedizin findet auch die Humanmedizin ihren Platz auf dem Portal und eine Frage und Sie erhalten innerhalb kurzer Zeit eine fundierte Antwort.

Auch das Abonnement einer Zeitschrift ist jetzt nur noch einen Mausklick entfernt: Bestellen Sie im "Shop" ein Zeitschriftenabo, kaufen Sie ein CME-Ticket oder entscheiden Sie sich gleich für das Online-Paket. So haben Sie Zugriff auf die gesamten Inhalte von springerzahnmedizin.de inklusive aller CME-Beiträge.

\section{Jetzt kostenfrei testen!}

Sie sind interessiert, möchten springerzahnmedizin.de aber erst einmal testen? Kein Problem! Registrieren Sie sich noch heute und nutzen Sie für 2 Monate den kostenfreien Testzugang zu springerzahnmedizin.de. 